\title{
Оценка температуры шнура тока, возникающего при переключении в халькогенидах системы GeSbTe
}

\author{
(C) С.А. Фефрелов ${ }^{1}$, Л.П. Казакова ${ }^{1,2}$, Н.А. Богословский ${ }^{1}$, К.Д. Цэндин ${ }^{1}$ \\ ${ }^{1}$ Физико-технический институт им. А.Ф. Иоффе Российской академии наук, \\ 194021 Санкт-Петербург, Россия \\ ${ }^{2}$ Санкт-Петербургский государственный лесотехнический университет им. С.М. Кирова, \\ 194021 Санкт-Петербург, Россия \\ E-mail: s.fefelov@list.ru
}

(Получена 7 июня в 2018 г. Принята к печати 18 июня 2018 г.)

Вольт-амперные характеристики тонкопленочных образцов системы GeSbTe измерены в режиме генератора тока. Исследованы осцилляции напряжения, наблюдающиеся после переключения. Показано, что эти осцилляции могут быть связаны с образованием горячего шнура тока и его постепенным остыванием. Сделаны оценки размера и температуры шнура тока. Показано, что характерная температура в шнуре тока соответствует температуре фазового перехода в кристаллическое состояние.

DOI: 10.21883/FTP.2018.12.46765.8931

\section{1. Введение}

В последнее время активно исследуются свойства халькогенидных стеклообразных полупроводников (ХCП) системы $\mathrm{Ge}-\mathrm{Sb}-\mathrm{Te}$. Благодаря низкой стеклообразующей способности и, как следствие, высокой скорости кристаллизации материалы этой системы используются в перезаписываемых оптических дисках $[1,2]$ и элементах энергонезависимой памяти с электрической записью информации $[3,4]$. Активно изучается влияние легирующих примесей на свойства $\mathrm{Ge}-\mathrm{Sb}-\mathrm{Te}$ [5-7]. К преимуществам материалов системы $\mathrm{Ge}-\mathrm{Sb}-\mathrm{Te}$ относятся высокая скорость записи, значительный оптический контраст и значительное различие сопротивлений кристаллической и аморфной фаз, низкое энергопотребление, а также хорошая масштабируемость.

Несмотря на значительные успехи в технологии, физика процессов, происходящих при электрической записи информации, остается предметом активных научных дискуссий. В частности, нет единого мнения о механизмах нелинейности ВАХ, заканчивающейся скачкообразным переходом в проводящее состояние (эффект переключения) [4].

В нашей работе [8] впервые была предложена методика измерения нелинейности и эффекта переключения в режиме заданного тока. В такой методике ток через образец не изменяется во время переключения, что позволяет разделить влияние электрического тока и напряжения. Кроме того, при измерениях в режиме заданного тока удалось наблюдать ряд интересных эффектов. Так, для некоторых образцов низкоомное состояние, образующееся после переключения, оказывалось неустойчивым, что приводило к возникновению колебаний напряжения на образце, связанных, по нашему мнению, с возникновением и постепенным исчезновением в образце области с высокой плотностью тока (шнура тока). Колебания напряжения на образце после переключения также наблюдались в работе [9], однако в этой работе наблюдались релаксационные колебания, связанные с зарядкой и разрядкой ёмкости, включенной в измерительную цепь параллельно исследуемому образцу. В нашей работе период колебаний как минимум на 2 порядка больше характерного времени $\mathrm{RC}$-колебаний, a сами колебания имеют постоянную амплитуду, следовательно, наблюдаемые колебания имеют принципиально другую природу.

В настоящей работе представлены результаты измерения ВАХ тонкопленочных образцов системы $\mathrm{Ge}-\mathrm{Sb}-\mathrm{Te}$ и по характеристикам колебаний напряжения сделаны оценки размера и температуры шнура тока. Полученные результаты хорошо согласуются с известными из литературы данными.

\section{2. Методика измерений и экспериментальные результаты}

Образцы для измерений представляли собой тонкопленочные структуры типа „сандвич“ из ХСП составов $\mathrm{Ge}_{2} \mathrm{Sb}_{2} \mathrm{Te}_{5}, \mathrm{Ge}_{15} \mathrm{Sb}_{15} \mathrm{Te}_{70}$ и $\mathrm{Ge}_{15} \mathrm{Sb}_{5} \mathrm{Te}_{80}$ и приготавливались методом термического напыления на стеклянные подложки с проводящим покрытием из $\mathrm{Au}$ или оксида индия-олова (ITO), которое служило нижним электродом. Верхний электрод - прижимной, точечный из золота. Площадь верхнего электрода составляла около $10^{-4} \mathrm{~cm}^{2}$. Для исследования ВАХ использовалась измерительная цепь с генератором тока $[10,11]$.

Проведение исследований в режиме генератора тока позволило детально исследовать ВАХ и процессы, происходящие при переключении и формировании состояния с памятью. Протекающий через образец ток $I$ формировался в виде импульса треугольной формы (рис. 1, кривая 1$)$. Ток в импульсе изменялся от 0 до $I_{\max }$. Максимальное значение тока $I_{\max }$ в различных измерениях устанавливалось в диапазоне 6.5 мкА-8.2 мА. 
При помощи цифрового осциллографа регистрировалось падение напряжения на образце $U$, величина которого определяется сопротивлением образца. Начальное сопротивление $R_{\text {init }}$ образцов в слабом поле (омическая область) определялось при помощи импульca с $I_{\max }=6.5$ мкА. Типичные значения $R_{\text {init }}$ лежали в диапазоне 100-300 кОм. При подаче импульсов с более высоким значением максимального тока наблюдалось переключение образца.

На рис. 1 приведена типичная форма напряжения на образце, наблюдаемая при переключении (кривая 2), которая характеризуется следующими параметрами: пороговое напряжение или напряжение переключения $U_{t h}$ соответствует переходу из высокоомного состояния в низкоомное; напряжение поддержки $U_{\text {hold }}$ соответствует участку с установившимся значением напряжения, на котором происходит формирование состояния с памятью.

Основными параметрами, характеризующими колебания напряжения на образце, являются период $\tau$, минимальное и максимальное значения напряжений $-U_{\min }$ и $U_{\max }$ соответственно, а также критическое значение тока $I_{c r}$ (рис. 1, кривая 1), ограничивающее область существования колебаний: при $I>I_{c r}$ колебания исчезают (срываются), при $I<I_{c r}$ колебания возможны. Типичная осциллограмма колебаний представлена на рис. 2.

Исследования показали, что для конкретного состава и толщины пленки величина периода колебаний достаточно стабильна и определяется током в образце и величиной напряжения пробоя активной области $U_{\max }$. При постоянном токе период практически не изменялся и не зависел от скорости нарастания тока в импульсе. Экспериментально установлено, что при увеличении тока через канал период уменьшается таким образом, что произведение периода на силу тока сохраняется (рис. 3).

Что касается амплитуды колебаний, то она при возрастании тока в образце практически не изменялась, вследствие чего значение пробивного напряжения $U_{\max }$ достигалось за меньшее время. При этом происходило уменьшение максимального сопротивления образца в области неустойчивости $R_{\max }$, которое вычислялось из данных колебательного процесса (рис. 2), по формуле $R_{\max }=U_{\max } / I$.

Как видно из рис. 2, колебания в области неустойчивости представляют собой последовательность переключений, характеризуемых амплитудой $U_{\max }$ и периодом $\tau$ на фоне монотонного изменения тока от 0 до $I_{\max }$. Рассмотрим отдельно взятый период, т.е. временной интервал $\tau$, в течение которого на образце происходит изменение напряжения от минимального $U_{\min }$ до максимального $U_{\max }$, а затем напряжение уменьшается от $U_{\max }$ до $U_{\min }$. Учитывая, что ток в образце на временно́м интервале $\tau$ меняется слабо, объяснить возрастание напряжения на образце от $U_{\min }$ до $U_{\max }$ возможно только возрастанием сопротивления шнура тока на этом интервале.

Также в работе было проведено измерение энергии активации проводимости исследуемых образцов в сла-

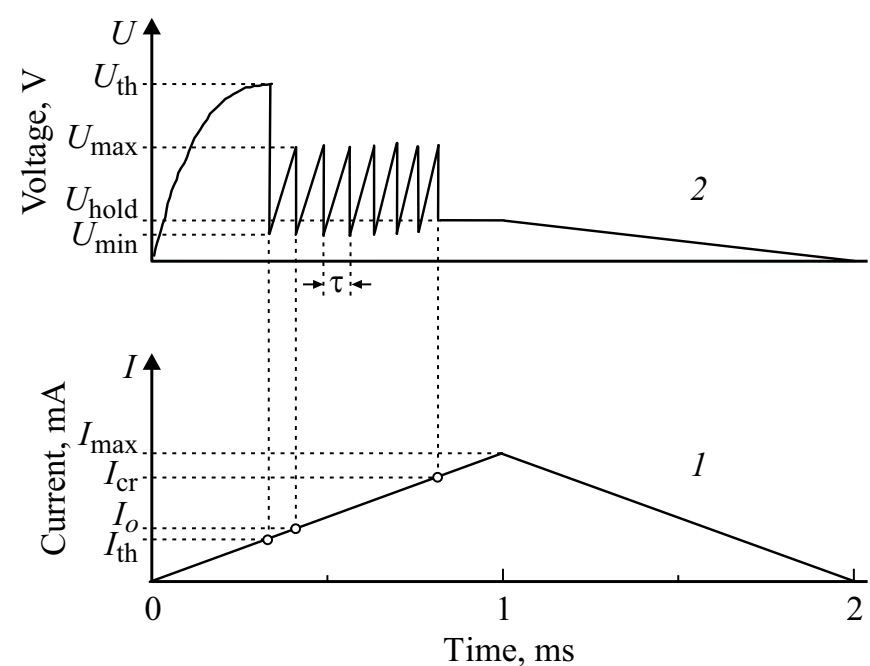

Рис. 1. Схематическое изображение осциллограмм тока (1) и напряжения (2) на образце.

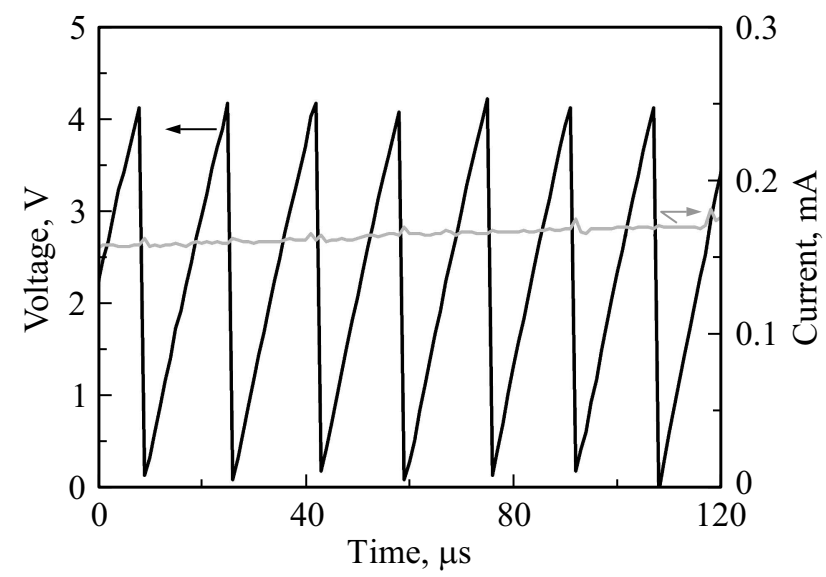

Рис. 2. Осциллограммы тока (1) и напряжения (2) на образце $\mathrm{Ge}_{15} \mathrm{Sb}_{15} \mathrm{Te}_{70}(d=700$ нм$)$ в области неустойчивости.

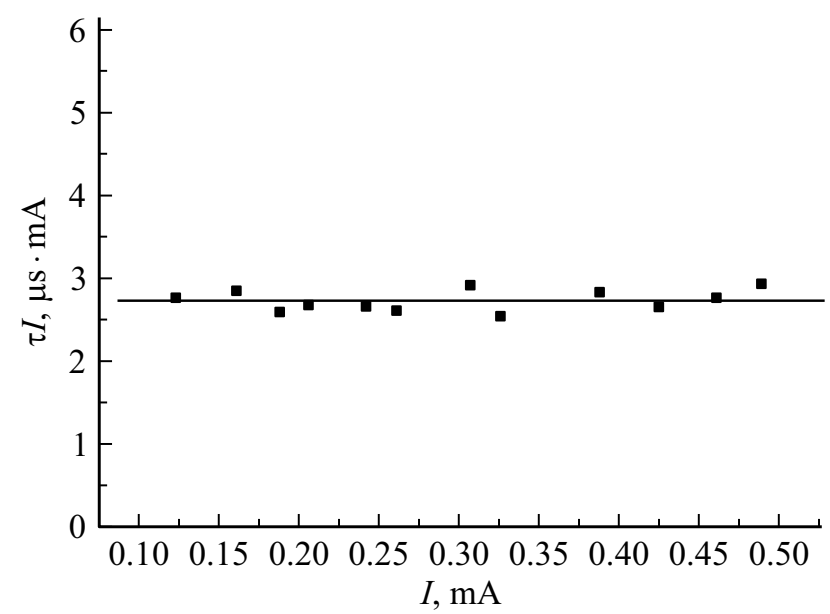

Рис. 3. Произведение периода $(\tau)$ на ток $(I)$ в зависимости от тока. 
бом электрическом поле. Для этого измерялась температурная зависимость начального сопротивления образцов $R_{\text {init }}$ в интервале температур $10-60^{\circ} \mathrm{C}$. При температурных измерениях образец помещался на модуль Пельтье. Датчиком температуры служил пленочный платиновый термометр сопротивления. В результате измерения было получено значение энергии активации $\Delta E=0.20 \pm 0.03$ эВ.

\section{3. Обсуждение результатов}

В работах [12-14] была предложена электроннотепловая модель, описывающая нелинейность ВАХ и эффект переключения в ХСП. В работе [15] в рамках этой модели было проведено численное моделирование процесса переключения. Показано, что при переключении в достаточно большом образце образуется шнур тока с высокой температурой. При этом время задержки переключения определяется в основном временем прогрева шнура. Поэтому в настоящей работе мы будем считать, что колебания напряжения на пленке связаны с образованием и постепенным рассасыванием горячего шнура тока.

Для оценки размеров шнура можно считать, что после переключения весь ток локализован в шнуре. Тогда, согласно данным рис. 2, сопротивление шнура порядка 1 кОм. Удельное сопротивление $\mathrm{Ge}_{15} \mathrm{Sb}_{15} \mathrm{Te}_{70}$ в проводящем состоянии можно оценить как $10^{-1} \mathrm{OM} \cdot \mathrm{cm}[16]$, соответственно площадь шнура тока составляет порядка $10^{-8} \mathrm{~cm}^{2}$, а радиус шнура тока $\sim 1$ мкм. Это значение согласуется с размерами шнура, полученными в работе [15] при помощи численного моделирования.

Переключение происходит очень быстро, поэтому выделением джоулева тепла в момент переключения можно пренебречь. Однако в процессе переключения происходит разрядка емкости системы, которая сопровождается выделением энергии в пленке. Полную емкость пленки и измерительной системы можно оценить как $C=40$ пФ. Напряжение на пленке перед переключением $U_{\max } \sim 4 \mathrm{~B}$, напряжение после переключения $U_{\min }$ мало. Тогда в процессе переключения в пленке выделяется тепло

$$
Q=\frac{C U_{\max }^{2}}{2} .
$$

Будем считать, что все это тепло идет на нагревание шнура тока с площадью сечения $S=10^{-8} \mathrm{~cm}^{2}$. Тогда изменение температуры шнура можно оценить как

$$
\Delta T=\frac{C U_{\max }^{2}}{2 \rho c S d},
$$

где $d-$ толщина пленки, $\rho$ и $c-$ удельное сопротивление и теплоемкость соответственно. Тогда для образца толщиной $d=600$ нм нагрев шнура можно оценить как $\Delta T \sim 300^{\circ} \mathrm{C}$.

Рассмотрим теперь процесс восстановления напряжения на образце после переключения. Сила тока через

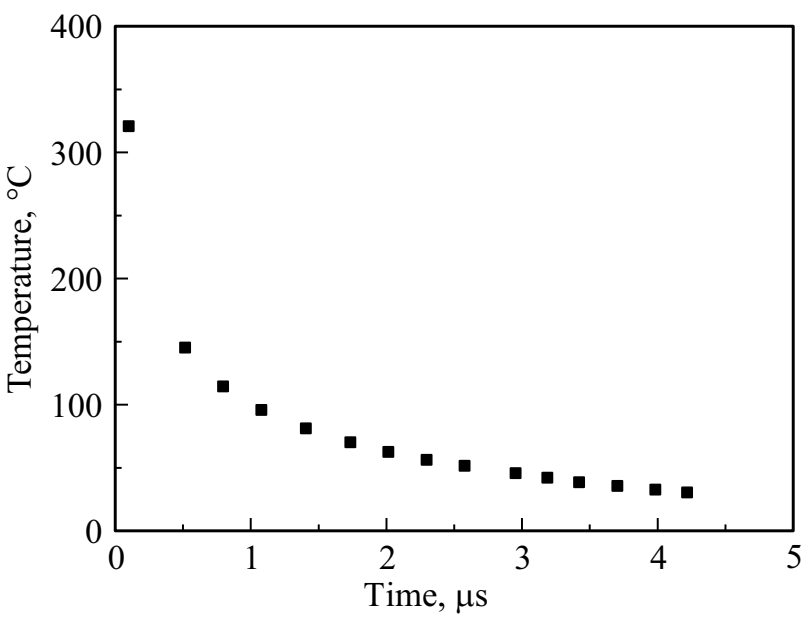

Рис. 4. Зависимость температуры в шнуре тока от времени.

образец остается постоянной, а напряжение возрастает, следовательно, возрастает и сопротивление образца. Логично предположить, что увеличение сопротивления связано с постепенным остыванием шнура тока. Следует обратить внимание на то, что диаметр шнура в наших образцах в разы больше длины шнура. Поэтому отвод тепла будет происходить преимущественно в контакты, а сам шнур при этом не будет значительно расширяться. Поэтому для оценки мы будем считать размеры шнура постоянными.

Поскольку после переключения напряжение на образце мало, влияние электронных процессов, таких как многофононная туннельная ионизация $U^{-}$-центров $[12,13]$, пренебрежимо мало. Тогда сопротивление шнура зависит от температуры активационным образом:

$$
R=R_{0} \exp \left(\frac{\Delta E}{k T}\right),
$$

где $\Delta E-$ энергия активации проводимости в слабом поле. Таким образом, по зависимости сопротивления шнура от времени можно восстановить зависимость температуры в шнуре от времени (рис. 4). При расчетах считалось, что температура при напряжении $U_{\max }$ равна $30^{\circ} \mathrm{C}$. В таком случае температура после переключения оказалась $\sim 320^{\circ} \mathrm{C}$, т. е. близка к температуре фазового перехода в кристаллическое состояние. Близкие значения температуры шнура были получены при других значениях тока через образец и соответственно других периодах колебаний.

Приведенные оценки подтверждают наше предположение о том, что колебания связаны с возникновением в образце горячего шнура тока и его последующим остыванием.

\section{4. Заключение}

В работе исследованы эффекты переключения и памяти в ХCП системы GeSbTe. Электрические измерения 
проведены в режиме заданного тока. На некоторых образцах после переключения наблюдались колебания напряжения на образце, связанные, по нашему мнению, с образованием и постепенным исчезновением шнура тока. При увеличении силы тока в образце период колебаний уменьшался. При этом установлено, что в широком диапазоне токов произведение силы тока и периода колебаний является постоянной величиной.

Проведены оценки размера и температуры шнура тока. Показано, что характерный радиус шнура в наших измерениях составляет $\sim 1$ мкм, характерная температура $\simeq 300^{\circ} \mathrm{C}$. Это значение близко к температуре фазового перехода в кристаллическое состояние, что согласуется с представлениями о том, что кристаллизация образца происходит в области с высокой температурой внутри шнура тока, образующегося после переключения.

\section{Список литературы}

[1] N. Yamada, E. Ohno, K. Nishiuchi, N. Akahira, M. Takao. J. Appl. Phys., 69 (5), 2849 (1991).

[2] M. Wuttig, N. Yamada. Nature Materials, 6, 824 (2007).

[3] G.W. Burr, M.J. Breitwisch, M. Franceschini, D. Garetto, K. Gopalakrishnan, B. Jackson, B. Kurdi, C. Lam, L.A. Lastras, A. Padilla, B. Rajendran, S. Raoux, R.S. Shenoy. J. Vac. Sci. Techn. B, 28 (2), 223 (2010).

[4] Н.А. Богословский, К.Д. Цэндин. ФТП, 46 (5), 577 (2012).

[5] O. Prkhodko, N. Almasov, S. Dyussembayev, S. Maksimova, V. Ushanov, K. Tsendin, S. Nesterov. Chalcogen. Lett., 10, 519 (2013).

[6] S. Raoux, M. Salinga, J.L. Jordan-Sweet, A. Kellock. J. Appl. Phys., 101, 044909 (2007).

[7] K. Darmawikarta, S. Raoux, P. Tchoulfian, T. Li, J.R. Abelson, S.G. Bishop. J. Appl. Phys., 112, 124907 (2012).

[8] N. Almasov, N. Bogoslovskiy, N. Korobova, S. Kozyukhin, S. Fefelov, L. Kazakova, S. Jakovlev, K. Tsendin, N. Guseinov. J. Non-Cryst. Sol., 358, 3299 (2012).

[9] M. Nardone, V.G. Karpov, I.V. Karpov. J. Appl. Phys., 107, 054519 (2010).

[10] С.А. Фефелов, Л.П. Казакова, С.А. Яковлев, С.А. Козюхин, К.Д. Цэндин. Тр. Междунар. симп. МФГФП-1 „Физика межфазных границ и фазовые переходы“ (Ростов н/Д, Россия, 2011) с. 155.

[11] С.А. Фефелов, Л.П. Казакова, С.А. Козюхин, К.Д. Цэндин, Д. Арсова, В. Памукчиева. ЖТФ, 84, 80 (2014).

[12] Н.А. Богословский, К.Д. Цэндин. ФТП, 43 (10), 1378 (2009).

[13] N.A. Bogoslovskij, K.D. Tsendin. J. Non-Cryst. Sol., 357, 992 (2011).

[14] N. Bogoslovskiy. Phys. Status Solidi B, 250 (8), 1563 (2013).

[15] N. Bogoslovskiy, K. Tsendin. Solid-State Electron., 129, 10 (2017).

[16] T. Kato, K. Tanaka. Jpn. J. Appl. Phys., 44 (10), 7340 (2005).

Редактор Г.А. Оганесян

\section{Estimation of the current filament temperature, which forms at switching in GeSbTe}

\author{
S.A. Fefelov ${ }^{1}$, L.P. Kazakova ${ }^{1,2}$, N.A. Bogoslovskiy ${ }^{1}$, \\ K.D. Tsendin ${ }^{1}$ \\ ${ }^{1}$ loffe Institute, \\ 194021 St. Petersburg, Russia \\ 2 St. Petersburg State Forest Technical University, \\ 194021 St. Petersburg, Russia
}

\begin{abstract}
The current-voltage characteristics of thin-film GeSbTe samples have been measured in the current control mode. Oscillations of the voltage on the sample after switching have been investigated. It is shown that these oscillations could be associated with the formation of a hot current filament and its gradual cooling. The size and temperature of the current filament have been estimated. The characteristic temperature of the current filament coincides with the temperature of the phase transition to the crystalline state.
\end{abstract}

